\title{
Ergonomic Advice Dissemination Method in the Immediate Postnatal Period: A One-Month Follow- Up Comparison of Verbal and Pamphlet-Based Advice on Pain Scores
}

\author{
Abha Dhupkar ${ }^{1}$, Sachita Gupta ${ }^{2}$ \\ ${ }^{1,2}$ D. E. Society’s Brijlal Jindal College of Physiotherapy, Pune, Maharashtra. \\ Corresponding Author: Abha Dhupkar
}

\begin{abstract}
Background: Pregnancy is a period where the musculoskeletal system undergoes various changes. These changes can cause stresses on the joints, leading to pain in them. Along with this, hormones like relaxin and oestrogen can cause laxity of ligaments and capsules, which can be another cause of strain on the joints. The pain patterns developed due to this can be carried to the post delivery period also. Ergonomics are explained in this period in order for the woman to function with less pain. The methods of reinforcing ergonomics are explored in this study.

Methods: 60 women were conveniently selected for this study. They were randomly assigned to verbal mode and pamphlet mode of ergonomic information dissemination at the start of the study. Pain was assessed at four points during the study: before, immediately after, a day after and a month after giving ergonomic advice.

Results: The Mann-Whitney U Test and repeated measures ANOVA were used to assess the pain levels. The between group pain and comfort levels showed no significant changes over the course of the study. The repeated measures ANOVA, which assessed within group responses, showed a significant change over the course of the study (p-value less than 0.0001).

Conclusion: Ergonomic information can be disseminated by verbal or pamphlet mode, the method can be decided as per the therapist's and the woman's need.
\end{abstract}

Key Words: ergonomic advice; ergonomic information; pamphlet; postnatal period.

\section{INTRODUCTION}

Pregnancy is a period where the body undergoes a variety of musculoskeletal, neurological, cardiovascular and hormonal changes 1,2,3. This period is mainly characterized by an increase in laxity of collagenous tissues ${ }^{1,2,3}$. The main reason for this increased laxity is to ease the process of actual birth of the foetus through the vaginal canal ${ }^{4,5}$.

The process of labour also marks an extra influx of the hormone relaxin, especially after the baby has crowned, in order to relax and increase the pelvic outlet, thereby increasing the cervical diameter and hence the vaginal diameter.

Laxity of ligaments is commonly seen in the pelvic joints ${ }^{5,6}$, mainly the sacro-iliac joint (SIJ). The SIJ, being a joint of minimal translatory movements 7 , can cause pain when the alignment of the joint is compromised during activities of daily living ${ }^{8}$. Along with this reason, many other causes of back pain can be seen during pregnancy.

One of the mechanisms of back pain is the abdominal distention throughout the duration of pregnancy. The physiology of this condition causes the uterus to increase 
in size, turning this pelvic organ into an abdominal one. As the uterine size increases in the later stages of pregnancy, the weight is borne more anteriorly by the woman. This causes the centre of gravity to shift anterior and upwards. The lumbar spine responds to the anterior loading of the body by increasing the lordotic curve of the lumbar section of the spine ${ }^{4,5,6}$. An extension moment occurs at the hip to counteract the weight of the foetus and the changed posture. This leads to an increased strain on the back, caused due to a change in the posture of the woman. The intervertebral discs are placed under increased strain due to these changes as well as in response to the hormonal influence of relaxin, leading to laxity of collagenous structures like ligaments and annulus fibrosus. These abnormal stresses placed on the joints of the low back, pelvis and hip lead to different pain patterns felt by the woman, commonly manifested as low back pain, pain in the SIJs, unilateral limb pain, pain when changing positions and frequently, pain which radiates down to one or both lower limbs. Many times, common movements like bearing weight on one limb can cause an increase in the pain felt by the woman $4,5,6$

Low back pain, during and after pregnancy, has been attributed to pelvic girdle pain and lumbar origin pain ${ }^{5}$. Lumbar origin pain occurs when lumbar joints, ligaments and discs are the sources of pain. Pelvic girdle pain occurs when the joints of the pelvic girdle are involved. Posteriorly the two SIJs and anteriorly the pubic symphysis, form the pelvic girdle.

Conservative management, consisting of physiotherapeutic exercises and pain management modalities, has been considered the mainstay of treatment for these conditions ${ }^{9,1-3,10,11}$.

Ergonomics can play a crucial role in conservative management, in the pregnancy period as well as post-delivery, to reduce pain and maintain an efficient functioning of the body ${ }^{1-3}$. The major reason to provide ergonomic advice in this period is to protect the body from injury. The woman is more prone to injuries in this period as the body has changed fundamentally. The ligament laxity cannot protect the joints when they overstrain. The weight increase also needs to be considered when performing daily activities and jobrelated tasks.

More importantly, the body does not return to its pre-pregnant state for 6 to 8 months after delivery. This period is more dangerous in terms of injury as the woman is expected to return to work and daily activities after a month or so. As the body is yet to revert to its original state, the woman can end up injuring herself if she does not take proper care in this duration. The injuries occurring in this period may not manifest immediately, they accumulate over the years and present in the early 50 s or late 40 s.

Ergonomic information related to the care to be taken in the post delivery period can be disseminated using either a verbal advice mode or a written mode in the form of a pamphlet. The efficacy of each mode yet needs to be established. The effective method of dissemination of ergonomic information can hence be established. This study, thus, aims to identify which method of information dissemination is the better method, with a view to decide the best possible method to provide information to patients.

\section{MATERIALS AND METHODS}

Ethical clearance was obtained from the institute. 60 women, who had delivered either vaginally or via LSCS, were chosen conveniently from the postnatal ward of a tertiary care hospital after obtaining their consent to participate in the study.

Two key criteria for inclusion in the study were an ability to read and write in Marathi and availability of a means of communication for follow-up.

All 60 women were given ergonomic advice verbally in the regional language (Marathi). Of these, 30 were randomly selected, using simple 
randomization, and given pamphlets written in the regional language (Marathi). Women with medical or surgical complications after delivery were excluded.

The mean duration of hospital stay after any type of delivery was 5 days at the hospital where the study was undertaken. Hence, women who had delivered their babies 5 or more than 5 days prior to receiving the advice were excluded.

The pamphlet consisted of ergonomic advice with pictorial representation of the same. The advice covered the following key areas:

1. Getting up from supine

2. Sitting postures

3. Breast feeding positions

4. Posture maintenance when performing daily activities

5. Techniques of lifting

6. Dos and don'ts to be followed.

The Numerical rating scale (NRS) was explained to the women for pain. Each woman reported pain on the NRS at four points in the study:

1. Before advice

2. Immediately after advice

3. One day after advice

4. One month after giving advice (reported via telephone)

The data was tabulated in Microsoft Excel. The responses were analyzed using the Mann-Whitney U test for between group analysis and the repeated measures ANOVA for within group analysis using GraphPad.

\section{RESULTS}

Of the women who participated in the study, 27 had delivered normally and 33 had delivered via LSCS. They were randomly allocated to either the pamphlet group or the verbal advice group. Their baseline parameters were comparable and no significant differences were noted in the women.

\section{DISCUSSION}

This study was performed to assess which method of ergonomic advice dissemination would be better in a clinical practice. The methodology, hence, reflected this by using a verbal and a written method of advice. As seen from the between group analyses before beginning the study, the women were comparable in their pain parameters. This denotes that no bias was present in the study at the beginning.

Ergonomic advice is very important in the period after delivery. The changes that have occurred during pregnancy have not normalized ${ }^{12-14}$ and the woman has to care for herself as well the baby in the post delivery period, most of the times starting immediately post-delivery. Also, the woman may be the sole carer in the family, thus, causing her to resume her household duties, without active support from other members of the family ${ }^{15}$.

Anecdotally, the woman is involved in standing for long durations for cooking; from half an hour to 2 hours, depending on the number of family members; has to get up repeatedly for feeding, changing, bathing the baby and in many households, prefers to clean the baby's and her clothes by herself, rather than depending on someone else for these tasks.

Activities carried out by the woman in the postpartum period can be affected if she has any residual pain related to the delivery process. Pelvic girdle pain persisting in the post-partum period can have debilitating effects on the woman. The pain and disability experienced due to this can cause her to reduce her activity levels and also can impact her ability to perform daily tasks ${ }^{16}$. This pain can occur due to a misalignment in the sacro-iliac joints or the pubic symphysis, perineal pain due to birthrelated injury or residual pain after a Caesarean section. These pain patterns can benefit from exercises and ergonomics. Hence, this period needs to be targeted for providing and ensuring the use of appropriate ergonomics.

The most often followed protocol after a delivery is to provide ergonomic advice and exercise only while the female is an inpatient. There are few instances of the female coming for a follow-up of either 
exercises or ergonomic advice in the early puerperal period. In such conditions, the advice to be given should be such that it is remembered by the female.

The use of verbal advice is a wellknown tool. A written/printed format was added to find if it was a better method to ensure ergonomic advice is followed. The results of the within group analysis for pain shows significant changes over the period of the study (Table 1).

Table 1: Table showing results of within group analysis for pain using repeated measures ANOVA.

Within group analysis of pain using repeated measures ANOVA.

\begin{tabular}{|l|l|l|}
\hline Item & With Pamphlet & Without Pamphlet \\
\hline Criterion & Pain measures & Pain \\
\hline Test & $\begin{array}{l}\text { Repeated measures } \\
\text { ANOVA Friedman test } \\
\text { ANOVA Friedman test }\end{array}$ \\
\hline P-value & $<0.0001$ & $<0.0001$ \\
\hline
\end{tabular}

Table 2:

Between Group analysis for Pain on NRS

Mann-Whitney U Test

\begin{tabular}{|l|l|l|l|l|l|}
\hline \multirow{2}{*}{ NRS } & \multicolumn{2}{|c|}{ Mean } & \multicolumn{2}{c|}{ SD } & \multirow{2}{*}{ P-value } \\
\cline { 2 - 5 } & A & B & A & B & \\
\hline Pre & 4.66 & 3.76 & 3.021 & 2.55 & 0.1513 \\
\hline Immediate & 4.56 & 3.76 & 2.95 & 2.55 & 0.1847 \\
\hline 1day & 4.03 & 3.56 & 2.69 & 2.38 & 0.3639 \\
\hline 1month & 0.16 & 0.5 & 0.53 & 0.97 & 0.1256 \\
\hline
\end{tabular}

Table showing results of between group analyses for pain using the Mann-Whitney $U$ test.

On considering individual analyses, the within group analysis (Table 2) for women who had only verbal advice shows a significant reduction in pain over a period of one month. Though change between pre and immediately post advice was not significant, change over a month was very significant. The same findings can be noted for the second group, who had a printed form of the advice along with verbal advice. This shows that either of the forms of advice can be used in the postnatal period.

Performing between group analyses to compare pain (Table 2) gave nonsignificant results. The main reason for this is the reduction in pain seen in both the groups. This underlines the fact that ergonomic advice can help in pain reduction. The method of providing this advice can be both verbal as well as a print form, with both methods showing similar results in pain reduction. This also underscores the need for ergonomics to be emphasized for this population.

Method of advice dissemination can alter the compliance of patient as well, though this was not a factor assessed in the present study. The woman was to follow ergonomics by herself for a period of one month without the therapist reminding her of the protocol. As this was a self-driven programme, compliance to the protocol was a factor that could have confounded the results. A limitation of this study was that the last data point was collected a month after the advice was given and no contact was maintained in this period to identify if the advice was being followed. A further scope to explore the efficacy of verbal and printed advice will be to maintain contact either every week or every two weeks till the collection of the last data point.

Limitations notwithstanding, ergonomic advice needs to be given to every woman in her pregnancy as well as after delivery. Back pain, due to its debilitating nature, can affect the quality of life in the postnatal period, especially if baby care is hampered due to it. For an early resumption of activities of self and baby care, ergonomics should be provided. This study underlines the need to spread awareness and information about ergonomics to help the woman improve her quality of life.

\section{CONCLUSION}

Use of ergonomics can reduce pain in a post-partum female. She should be encouraged to follow joint protection techniques. The advice can be given in print form preferentially to reinforce the verbal advice.

Ethical Clearance: Institution level.

Sources of Funding Support: None declared.

Conflict of Interest: None declared.

\section{Acknowledgements}

We thank all the study participants for their enthusiastic participation in the study. 


\section{REFERENCES}

1. Women's Health: a textbook for physiotherapists. Ruth Sapsford, Joanne Bullock-Saxton, Sue Markwell. 1st ed.; Elsevier, 1997.

2. Physiotherapy in Obstetrics and Gynaecology. Margaret Polden, Jill Mantle. 1st ed.; Butterworth- Heinemann, 1990.

3. Physiotherapy in Obstetrics and Gynaecology. Jill Mantle, Jeanette Haslam, Sue Barton. 2nd ed.; ButterworthHeinemann, 2004.

4. Myles MacEvilly, Donal Buggy. Back pain and pregnancy: a review. Pain; 1996; 64: 405-414.

5. Katonis $\mathrm{P}$, Kamppouroglou $\mathrm{A}$ et al. Pregnancy-related low back pain. Hippokratia; (2011); 15 (3), 205-210.

6. Jennifer Sabino, Jonathan N Grauer. Pregnancy and low back pain. Curr Rev Musculoskelet Med; (2008); 1:137-141.

7. The physiology of the joints. I.A. Kapandji. 2nd ed; Churchill Livingstone, 1970.

8. Damen L, Buyruk HM et al. Pelvic pain during pregnancy is associated with asymmetric laxity of the sacroiliac joints. Acta Obstet Gynecol Scand; 2001; 80: 1019-24.

9. Tseng et al. A systematic review of randomised controlled trials on the effectiveness of exercise programs on lumbopelvic pain among postnatal women. BMC Pregnancy and Childbirth; (2015); 15:316-328.

10. G A Dumas, J G Reid et al. Exercise, posture and back pain during pregnancy.
Clinical Biomechanics; (1995); Vol 10, No 2 98-103.

11. G A Dumas, J G Reid et al. Exercise, posture and back pain during pregnancy Part 2: Exercise and back pain. Clinical Biomechanics; (1995); Vol 10, No 2 104109.

12. Mattea Romano, Alessandra Cacciatore et al. Postpartum period: three distinct but continuous phases. Journal of Prenatal Medicine; 2010; 4(2): 22-25

13. Pat McGovern, Bryan Dowd et al. Postpartum health of employed mothers 5 weeks after childbirth. Ann Fam Med; 2006; 4: 159-167.

14. Pat McGovern, Bryan Dowd et al. Mothers' health and work-related factors at 11 weeks postpartum. Ann Fam Med; 2007; 5: 519527.

15. Merlyn Rodrigues, Vikram Patel et al. Listening to mothers: qualitative studies on motherhood and depression from Goa, India. Social Science and Medicine; 2003; 57: 2797-1806.

16. Jorun Engeset, Britt Stuge, Liv Fegran. Pelvic girdle pain affects the whole life - a qualitative interview study in Norway on women's experiences with pelvic girdle pain after delivery. BMC Research Notes; 2014; 7: 686-693.

How to cite this article: Dhupkar A, Gupta S. Ergonomic advice dissemination method in the immediate postnatal period: a one-month follow-up comparison of verbal and pamphletbased advice on pain scores. Int $J$ Health Sci Res. 2021; 11(9): 265-269. DOI: https://doi. org/10.52403/ijhsr.20210940 